\title{
The role of iron regulatory proteins in the control of iron metabolism in mammals
}

\author{
Agnieszka Styś, Rafae R. Starzyński, PaWee LiPińSki ${ }^{*}$ \\ Department of Molecular Biology, Institute of Genetics and Animal Breeding \\ Polish Academy of Sciences, Jastrzębiec, Poland \\ *Corresponding author: p.lipinski@ighz.pl
}

\begin{abstract}
The iron regulatory proteins (IRP1 and IRP2) are two cytoplasmic RNA-binding proteins involved in the mechanisms that control iron metabolism in mammalian cells. They modulate the expression of iron-related proteins at a post-transcriptional level by binding to specific iron regulatory elements (IREs) on their mRNAs. IRP-IRE interaction can block protein synthesis or stabilize the mRNA. At low intracellular iron concentration, IRPs bind to the IRE of ferritin or ferroportin mRNAs and block their translation. Direct interactions between IRPs and several IRE motifs stabilize transferrin receptor mRNA. The converse regulation of ferritin and TfR synthesis, being a consequence of the lack of binding of IRPs to IRE, occurs in cells with high iron level. Thus, IRP-mediated regulation rapidly restores the physiological level of iron during its deficiency as well as excess. The role of IRPs in maintaining the intracelluar iron balance has been relatively well characterized in numerous types of mammalian cells. However, the importance of IRPs in the regulation of systemic iron metabolism in mammals, particularly, in signaling between the cells which play major roles in body iron metabolism, such as duodenal enterocytes, reticuloendothelial macrophages, hepatocytes, and bone marrow precursors of red blood cells, is only beginning to be investigated. Several studies have shown that IRP2 is a predominant regulator of iron homeostasis in mice housed under standard conditions, thus limiting the impact of IRP1 on this metabolic pathway. Although IRP1-deficient mice do not display a strong pathological phenotype, a deletion of both IRPs is embryonic lethal. In addition, in vitro and in vivo studies have reported that nitric oxide (NO) and hydrogen peroxide $\left(\mathrm{H}_{2} \mathrm{O}_{2}\right)$, which are produced during inflammation, are potent IRP1 regulators that mediate the disassembly of Fe-S cluster of IRP1. There is also an increasing evidence that $\mathrm{NO}$ and superoxide anion $\left(\mathrm{O}_{2}{ }^{--}\right)$may induce a strong down-regulation of IRP1 at the protein level and thus have an impact on the binding of IRP1 to IREs. All these data suggest a predominant role of IRP1 in the regulation of iron homeostasis under specific physiopathological conditions.
\end{abstract}

Key words: iron regulatory, IRP, IRE, iron homeostasis

\section{Introduction}

Iron is an extraordinary microelement because its redox properties make it essential for the basic biological processes in almost all living organisms - except for Lactobacillus bacteria (Weinberg, 1997). Interestingly, the same properties also make iron toxic. This metal is found in active sites of a large number of enzymes required for key life processes, such as oxygen transport, electron transfer and DNA synthesis. At the same time, iron is toxic due to its capacity to catalyse the production of reactive oxygen species which promote oxidative damage to vital cellular structures. In numerous diseases, the common pathway leading to tissue damage is the iron catalyzed Haber-Weiss reaction, in which the relatively harmless oxygen products: superoxide $\left(\mathrm{O}_{2}^{-{ }^{-}}\right)$ and hydrogen peroxide $\left(\mathrm{H}_{2} \mathrm{O}_{2}\right)$ are transformed into the highly toxic hydroxyl radical ( $\mathrm{OH}$ ) (Bartosz, 2003). In a solution, iron can exist in two oxidation states: Fe(II) and $\mathrm{Fe}(\mathrm{III})$. However, at physiological pH, $\mathrm{Fe}$ (III) form is very poorly soluble. The above-mentioned iron properties impose the functional duality of its homeostasis, i.e. the maintenance of iron bioavailability and the limitation of iron toxicity. Many proteins have evolved to carry iron in biological fluids between cells, to transport it through cellular membranes and to store it in a non-toxic, but easily mobilisable form.

A considerable number of proteins involved in iron homeostasis have been identified and characterized within 
the last 15 years. Similarly, recent researches have revealed the identity of complex regulatory mechanisms that control iron metabolism (Andrews, 2008). In particular, regarding systemic iron balance, a regulatory axis between the liver-derived peptide hepcidin and the iron exporter ferroportin proved to be fundamental for the coordination of iron fluctuations in the body and its distribution among the main sites of iron metabolism such as absorptive enterocytes, reticuloendothelial macrophages, hepatocytes and erythroid precursors of red blood cells. Hepcidin inhibits the cellular iron efflux by binding to ferroportin, the only known cellular iron exporter, and inducing its subsequent degradation. Inappropriately low hepcidin levels cause iron overload disorder - hemochromatosis - whereas increased hepcidin expression leads to a diminished intestinal iron absorption and iron arrest in the reticuloendothelial system, leading to functional anemia. For more details, we recommend recent comprehensive reviews (Andrews, 2008; Hentze et al., 2010; Viatte and Vaulont, 2009).

In contrast to the quite recent advances in the studies of systemic iron regulation, the molecular basis of cellular iron management through iron-regulatory proteins (IRP1 and IRP2) that bind iron-responsive elements (IREs) in regulated messenger RNAs have been described in 1980s (Leibold and Munro, 1988; Mullner and Kuhn, 1988). Role of IRPs in the maintenance of intracelluar iron balance has been well characterized in numerous types of immortalized mammalian cell lines. However, the physiological importance of (IRE/IRP) regulatory network in the regulation of iron metabolism for in vivo mammalian models is only beginning to be investigated. Similarly, IRP specificity and/or redundancy in the regulation of intracellular iron in response to different stimuli has become a hot topic (Lipiński and Starzyński, 2006; Muckenthaler et al., 2008).

\section{A brief outline of a cellular iron metabolism}

All aspects of intracellular iron homeostasis are mirrored in the so-called labile iron pool (LIP; also referred to as chelatable iron pool CIP), a low-molecular-weight pool of weakly chelated iron in the cytosol that rapidly transits through the cell becoming a source of iron for heme synthesis and iron-sulfur cluster biogenesis. It has been claimed that LIP is also a cellular source of iron participating in the Fenton reaction, as well as a sensor for the IRE/IRP regulatory mechanism. Thus, LIP consti- tutes a real crossroads of cellular iron metabolic pathways. It seems that under physiological conditions, a LIP level is a midway between the cellular need for iron and the hazard of excessive generation of ${ }^{\circ} \mathrm{OH}$ radical. Iron concentration in LIP is primarily determined by the rate of iron uptake, storage and release, mostly accomplished by three proteins, transferrin receptor 1 , ferritin and ferroportin, respectively (Kakhlon and Cabantchik, 2002; Kruszewski, 2003).

Iron bound to transferrin (Tf) enters the cells viacell surface TfR1-mediated endocytosis to form clathrin vesicle which matures to an ATP-dependent proton-pumping endosome. Here, the proton influx lowers $\mathrm{pH}$ to approximately 5.5 , and subsequently induces iron release from Tf. Apo-Tf still remains bound to its receptor at this acidic $\mathrm{pH}$. The transport of iron liberated from Tf across the endosomal membrane to cytosol is mediated by divalent metal transporter 1 (DMT1), whereas the apoTf-TfR1 complex is recycled back to the cell surface. In the neutral $\mathrm{pH}$ of extracellular environment, apo-Tf dissociates from its receptor (Feelders et al., 1999).

Iron in excess of metabolic needs is stored in a soluble and non-toxic form inside ferritin (Ft). Ft is a ubiquitous protein composed of a protein shell that can accommodate up to $\sim 4500$ atoms of iron. It is a heteropolymer composed of 24 subunits of two types: L (L-Ft for light) and $\mathrm{H}$ (H-Ft for heavy), depending on their relative molecular weights (in most mammalian species, except from mouse). The two subunit types have different functional properties. The $\mathrm{H}$ chain has an iron-binding site, where $\mathrm{Fe}(\mathrm{II})$ is oxidized to $\mathrm{Fe}(\mathrm{III})$, whereas $\mathrm{L}$ chain, which does not exhibit ferrooxidase activity, has a nucleation site involved in iron-core formation (Arosio and Levi, 2010). Recently, a cytosolic iron chaperone PCBP1 that can be involved in the delivery of iron from LIP to ferritin has been described (Shi et al., 2008). Physiological factors releasing iron from ferritin have not been identified thus far. It seems that lysosomal degradation of ferritin serves as the main route of iron release from this protein (Radisky and Kaplan, 1998).

The process of iron transport to an extracellular environment involves the only known mammalian iron exporter i.e., ferroportin (Fpn) that is expressed at the cell surface. The export of ferrous iron by Fpn is concomitant with its oxidation to the ferric state by proteins from the multi-copper oxidase family, ceruloplasmin and hephaestin (Ganz, 2005). 


\section{The IRE/IRP regulatory system}

The cellular iron homeostasis is largely controlled by the post-transcriptional IRE/IRP regulatory system. Iron regulatory proteins (IRP1 and IRP2) are cytoplasmic proteins that play a critical role in this regulation by interacting with mRNA hairpin structures called iron responsive elements (IREs). These elements, showing an evolutionarily conserved 28 nucleotide sequence, (for details, see Figure 1) are present in the untranslated regions (UTR) of mRNAs encoding subunits of iron storage protein, ferritin and in both iron transporters [transferrin receptor 1, (TfR1) and ferroportin, (Fpn)] involved in iron import and export, respectively (Wallander et al., 2006). Although the presence of IREs has also been identified in several other mRNAs including those encoding proteins involved in heme synthesis in erythroid cells (eALAS) (Dandekar et al., 1991); iron uptake (DMT1) (Gunshin, 1997) as well as two tricarboxylic acid cycle enzymes [mitochondrial aconitase (Zheng, 1992) and the subunit of succinate dehydrogenase (Kohler et al., 1995)], their functional consequences have not been well defined yet. The binding of IRPs to the unique IRE in the 5'-UTR of L- and H-Ft mRNAs blocks the translation initiation by preventing the association of $43 \mathrm{~S}$ translation pre-initiation complex. In fact, the recruitment of a small ribosomal subunit by the cap-binding complex eIF $4 \mathrm{~F}$ is prevented, probably due to the steric hindrance (Muckenthaler et al., 1998). In contrast, the binding of IRPs to IREs in the 3'-UTR of TfR1 mRNA is thought to protect this mRNA against degradation by preventing access of a nuclease whose cleavage site is close to the IREs (Binder et al., 1994). The coordinated bidirectional regulation of Ft, TfR1 and Fpn mRNAs by IRPs (depending on IRE localization) allows rapid changes in gene expression in response to iron fluctuations, and ensures that the cells acquire sufficient iron for their requirement while preventing iron toxicity.

IRP1 is a bifunctional protein showing either aconitase or trans-regulatory activity. Importantly, both IRP1 activities are mutually exclusive depending on the presence or absence of the [4Fe-4S] cluster (Volz, 2008). The crystal structure of human IRP1 in its aconitase form shows evidence that IRP1 is a four-domain protein, with domains 1-3 linked to domain 4 by flexible hinge linker peptide (Dupuy et al., 2005). In iron-replete cells, IRP1 assembles an iron-sulfur [4Fe-4S] cluster in the cleft between domains 1-3 and domain 4 and functions as a cytosolic aconitase that catalyzes the stereo-specific conversion of citrate to isocitrate via the intermediate cisaconitate. The [4Fe-4S] cluster of IRP1 is inserted into its active site. In this "closed" conformation, the IRE-binding site is not accessible. Three of the iron atoms of the cluster are ligated to three cysteine residues (cys437, cys503 and cys506), whereas the fourth iron, termed a labile iron, lacks protein ligand, interacts with citrate, and enables its conversion to isocitrate. A mutation in any of these three cysteines was shown to result in a complete loss of IRP1 aconitase activity (Hirling et al., 1994).

Under iron-deficient conditions, IRP1 accumulates as an apo-form, lacking the [4Fe-4S] cluster, and gains the ability to recognize IREs with high affinity. In this situation, IRP1 is considered to have an "open" conformation with the accessible IRE-binding site. A detailed list of IRP1 residues involved in, or affected by, IRE binding has been shown by Dupuy et al. (Dupuy et al., 2005). Among them, three cysteines predicted to coordinate the [4Fe-4S] cluster in IRP1 molecule were also found to be indispensable for IRE-binding, which confirms that iron-sulfur cluster assembled within IRP1 excludes the binding to the IRE.

The mechanism by which cellular iron availability regulates the insertion or removal of the $[4 \mathrm{Fe}-4 \mathrm{~S}]$ cluster remains unknown. The reactivation of IRE-binding following iron depletion is a slow process that takes approximately 15 hours (it is thought to correspond to the accumulation of neo-synthesized apo-IRP1) while inactivation of IRP1 IRE-binding activity by high iron levels seems to occur rapidly (2-4 hours) (Wallander et al., 2006). The biosynthesis of iron-sulfur clusters in eukaryotes and their subsequent insertion into target apo-proteins is a complex process taking place in the cytosol and mitochondria and involving more than 20 proteins (Ye and Rouault, 2010). However, little is known about the cellular factors that directly influence the insertion of iron sulfur cluster to apo-IRP1. There is evidence from recent experiments using IRP1 and IRP2-deficient hepatocytes that IRP1 plays a major role in adequate iron supply to the mitochondrion, which is indispensable for undisturbed biogenesis of iron-sulfur clusters (Galy et al., 2010). It is therefore possible that IRP1 may constitute an important element of auto-regulatory loop determining its activities. 


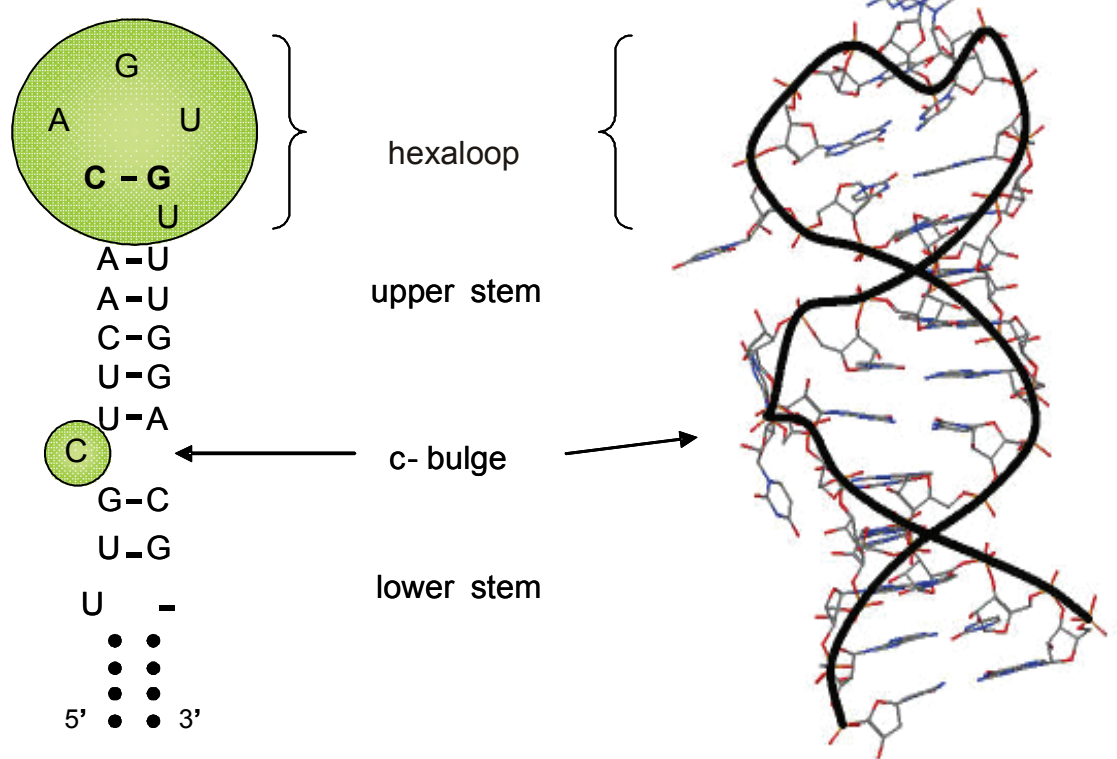

Fig. 1. H- Ferritin IRE structure. IREs are conservative hairpin structures located in the untranslated regions of mRNAs encoding proteins important for iron metabolism, e.g. H-ferritin. Adapted from (Zheng et al., 1992)

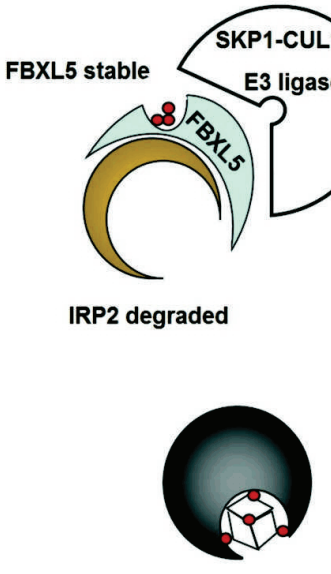

IRP1 aconitase high iron
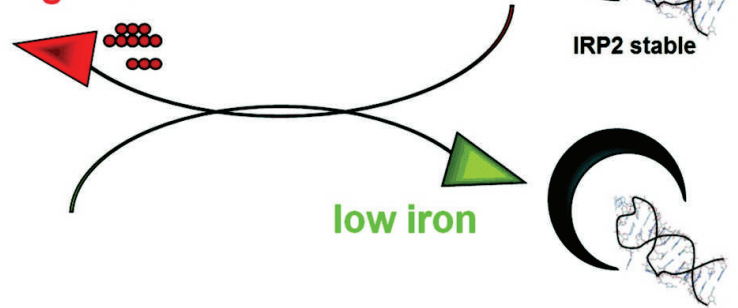

IRP1 binds IRE

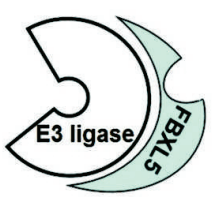

FBXL5 degraded

Fig. 2. A model of IRP1 and IRP2 regulation by fluctuations in iron concentration. In iron-replete cells, IRP1 assembles an ironsulfur cluster and functions as a cytosolic aconitase. In this 'closed' conformation, the IRE-binding site is not accessible. IRP2 is targeted by E3 ubiquitin ligase complex containing the FBXL5 for proteasomal degradation. In this complex, FBXL5 serves as an iron sensor that is stabilized under iron-replete conditions and degraded upon iron depletion. Under iron-deficient conditions, IRP1 accumulates as an apo-form, lacking the [4Fe-4S] cluster, and gains the ability to recognize IREs with high affinity and IRP2 is stabilized

IRP2 was shown to specifically interact with the IRE localized in ferritin subunits mRNA and with an affinity as high as that of IRP1 (Henderson et al., 1994; Guo et al., 1994; Samaniego et al., 1994). IRP2 also binds with a similar affinity as IRP1 to the IREs present in the TfR1 mRNA (Kim et al., 2004; Kohler et al., 1995). In spite of its $61 \%$ sequence identity with IRP1, IRP2 does not possess iron-sulfur cluster and does not exhibit aconitase activity, although it conserves the cysteines of the active site of IRP1 (cys512, cys578, cys581) (Guo et al., 1995). Unlike IRP1, changes in the IRE binding activity of IRP2 being a consequence of alterations in iron availability, do 
not result from a post-translational protein activity conversion (Kim et al., 2004). IRP2 is regulated at the protein stability level, namely it is stabilized when iron is scarce and targeted to degradation by the proteasome in iron replete-cells. IRP2 differs from IRP1 by the insertion of a 73 amino acid domain near its amino terminus (Guo et al., 1995). The domain is rich in cysteines, serines and prolines and contains a site susceptible for proteolysis (Guo et al., 1995). Previous reports showed contradictory results regarding the role of unique 73aminoacid region of IRP2 as a substrate for the haemoxidized IRP2 ubiquitin ligase (HOIL-1) in the iron-dependent degradation of IRP2 (Ishikawa et al., 2005; Hanson et al., 2003). Recently, two groups proposed that E3 ubiquitin ligase complex containing the FBXL5 protein targets IRP2 for proteasomal degradation. The stability of FBXL5 itself is regulated by iron, in the opposite direction of IRP2, namely it is stabilized under ironreplete conditions and degraded upon iron depletion (Salahudeen et al., 2009; Vashisht et al., 2009). Thus, FBXL5 with a domain that binds iron acts as a sensor to control iron metabolism in human cells.

In summary, in iron-replete cells, concerted changes in IRPs activities, i.e., low IRE-binding activity of both IRPs, lead to the down-regulation of TfR1, up-regulation of L-Ft, H-Ft and Fpn, thereby decreasing cellular iron uptake and increasing both storage and export of intracellular iron, respectively. In iron deficiency, a converse regulation takes place, which is mediated by high IREbinding activity of both apo-IRP1 and stabilized IRP2. The resulting elevated iron uptake and limited iron storage and export allow the restoration of LIP levels and iron homeostasis maintenance.

\section{The role of IRPs in mammalian physiology}

IRP1 and IRP2 are ubiquitously expressed in all mammalian tissues. In general, IRP1 is more abundant than IRP2. In mice, IRP1 protein levels are highest in the kidney, brown fat and liver whereas IRP2 expression predominates in several brain regions such as forebrain and cerebellum (Meyron-Holtz et al., 2004). Physiological significance of the occurrence of the two ubiquitously expressed IRPs, with similar affinity for IREs and equal ability to regulate $\mathrm{Ft}$ emerges as an intriguing topic in the field of IRP biology. Functional redundancy is undoubtedly one of the possible explanations of having two IRPs. However, possible specificity of IRPs in res- ponse to various stimuli may be associated with distinct mechanisms by which IRPs IRE-binding activity is regulated (IRP1: by an iron-sulfur switch; IRP2: efficient degradation in an iron-dependent manner).

Generation of mouse models of IRP deficiency (Galy et al., 2004; Galy et al., 2005; LaVaute et al., 2001; Meyron-Holtz et al., 2004) has greatly increased our knowledge on the role of these regulators in iron metabolism. IRP1- and IRP2-deficient mice have become a powerful model to investigate the mechanisms that regulate iron homeostasis and to determine the physiological role of each IRP. An early lethality of the mouse embryos lacking IRP1 and IRP2 underlines the crucial role of the IRP/IRE regulatory system in the regulation of iron metabolism at initial stages of murine embryonic development (Smith et al., 2006). Moreover, this also suggests that IRP1 and IRP2 have overlapping functions.

The evidence of IRPs redundancy also derives from the fact that the neurodegeneration and anemia of IRP2deficient mice is greatly exacerbated in animals that also lack one copy of Aco1 gene (encoding IRP1) (Smith et al., 2004). The misregulation of the systemic iron metabolism in mice, lacking both IRPs in the small intestine, in a tissue-specific manner, [generated using Cre/Lox technology (Nagy, 2000)] clearly showed that the duodenum plays a crucial role in systemic iron homeostasis and that IRPs are essential for the control of key iron absorption molecules in the duodenum (Galy et al., 2008). Overall targeted deletions of IRP1 and IRP2 in mice have demonstrated that IRP2 is the main iron sensor and cellular regulator under basal conditions. Mice deficient in IRP1 do not significantly misregulate iron metabolism (Galy et al., 2005; Meyron-Holtz et al., 2004), except in kidney and brown fat, in which IRP1 expression and the ratio between IRP1 and IRP2 is high (Lipiński et al., 2005). This is consistent with the previous report that IRP1 is not required for normal regulation of IRP targets in a lymphocyte cell line (Schalinske et al., 1997). Furthermore, it is worth recalling that in quiescent cells, bifunctional IRP1 is mostly a [4Fe-4S] cluster aconitase which does not influence iron metabolism. This partly explains why IRP1-deficient mice display only a mild iron phenotype. In contrast, in mice with a targeted deletion of IRP2 (IRP2-deficient, IRP2 - null mice), most tissues exhibit iron misregulation symptoms and the mice develop microcytic anemia (Galy et al., 2005; LaVaute et al., 2001) and a progressive neuro- 
degenerative disease (LaVaute et al., 2001). The main molecular hallmark of IRP2 deficiency is the over-expression of ferritin and insufficient expression of TfR1, leading to cellular iron depletion, which disturbs the functions of many cell types, in particular, erythroid cells known for extensive heme synthesis. In the absence of IRP2, IRP1 IRE-binding activity does not increase, whereas in the absence of IRP1, IRP2 shows a compensatory increase both at protein and RNA-binding activity levels. Importantly, in mice fed with low-iron diet, IRP1 does not convert to the cluster-free apoform whereas IRP2 is stabilized (Meyron-Holtz et al., 2004). This suggests that IRP2 is also the main regulator of iron metabolism in response to fluctuations in iron concentration.

\section{Does IRP1 dominate cellular iron metabolism regulation in response to nitric oxide?}

Nitric oxide (NO) is a short-life molecule produced by the enzyme known as the nitric oxide synthase (NOS), in a reaction that converts arginine and oxygen into citrulline and NO. There are three isoforms of the enzyme i.e., neuronal NOS (also called NOS1), inducible NOS (iNOS or NOS2), and endothelial NOS (NOS3). The 'simple gas' nitric oxide plays a ubiquitous role in the body, from maintaining vascular homeostasis and fighting infections to acting as a neurotransmitter. $\mathrm{NO}$ also appears to be an important signaling and effector molecule that is able to widely affect intracellular iron metabolism (Watts et al., 2003). Modulation of the IRP1 function is an important element in the complex network of interactions between NO and intracellular iron (reviewed in (Bouton and Drapier, 2003). Indeed, apart from the regulation mediated by iron availability, $\mathrm{NO}$ was the first physiological molecule found to be able to convert IRP1 from aconitase to the IRE-binding form in mammalian cells (Drapier et al., 1993; Weiss et al., 1993). A sustained NO production generally occurs during inflammatory settings where macrophages exhibit high NO synthase 2 expression. Once released, NO directly targets the [4Fe-4S] cluster of IRP1, promoting its gradual disassembly and complete removal, thus favoring the IREbinding conformation of the protein (shifting IRP1 to its IRE-binding form) (Bouton et al., 1996; Soum and Drapier, 2003). Subsequently, IRP1 tightly binds to IRE and exerts its iron-regulatory function. Accordingly, it has been established that endogenous NO production as well as the exposure of cells to exogenous NO was capable of repressing ferritin mRNA translation, stabilizing transferrin receptor mRNA and, in consequence, increasing LIP levels (Lipiński et al., 2005). When NO flux stops, the process is reversed: the IRP1 molecules readily dissociate from IRE and regain aconitase activity after reassembling an [4Fe-4S] cluster. Importantly, the dissociation of IRP1 from the IRE followed by Fe-S cluster repair was accompanied by the restoration of the basal Ft levels (Bouton et al., 2002). In the above-mentioned context NO proves to be an authentic regulator.

Besides IRP1, IRP2 has also been shown to be regulated by NO. The mechanism of NO action on IRP2 activity has long been studied in various cell types and different NO sources. However, the conclusions reached by many groups are seemingly contradictory, ranging from the lack of regulation in response to NO (Bouton et al., 1998; Mulero and Brock, 1999; Phillips et al., 1996), to NO-dependent IRP2 degradation (Kim et al., 2004; Recalcati et al., 1998), and conversely to NO-dependent upregulation of IRP2 (Wang et al., 2005). These discrepancies led to difficulties in revealing the intrinsic contribution of each IRP in the regulation of cellular iron metabolism by NO.

Interestingly, IRP1- and IRP2-deficient mice have proved to be again an irreplaceable experimental model to disclose these discrepancies. Experiments using IRP1- and IRP2-knockout mice were undertaken in our laboratory to discriminate the role of each IRP in the regulation of iron metabolism by $\mathrm{NO}$ in bone marrowderived macrophages. Our results clearly show that IRP1 is predominant in controlling iron metabolism in response to $\mathrm{NO}$ derived from the slowly-releasing NOdonor known to mimic long-lasting NO delivery during inflammation (Styś et al., 2010). The reason for this domination is likely to be intimately related to the fact that IRP1 detects NO through the disassembly of its iron-sulfur cluster. This allows IRP1 to switch to an IREbinding protein, which then mediates the regulation of cellular iron metabolism at the expense of IRP2.

Most studies on the regulation of IRP1 activities have been focused on post-translational mechanisms underlying the insertion/extrusion of an [4Fe-4S] cluster into/ from the IRP1 molecule. However, NO was also shown to down-regulate the expression of Aco1 gene leading to the decrease in intracellular IRP1 protein levels (Oliveira and Drapier, 2000). Mechanism of this regulation involves NO-dependent degradation of STAT5 proteins, 
which inhibits the expression of the Acol gene at the transcriptional level (Starzyński et al., 2006). Biological importance of this regulation may be the restriction of NO-dependent IRP1 activation and, consequently, the attenuation of the NO-induced rise in the LIP, a major factor exacerbating oxidative injury.

\section{Multidirectional regulation of IRP1 by reactive oxygen species}

Iron and oxygen $\left(\mathrm{O}_{2}\right)$ are intimately associated in many well-characterized patho-physiological processes. It is generally believed that the generation of $\mathrm{OH}$, a highly destructive oxidant, through the Fenton reaction is the main point at which the pathologic interaction between the oxygen and the iron occurs. Since no specific molecule has evolved to fulfill the role of ${ }^{\circ} \mathrm{OH}$ scavenger, living organisms have developed different ways to prevent its formation including the meticulous control of iron metabolism. As exemplified by the comparison of iron metabolism in the two lines of mouse lymphoma L5178Y cells differentially sensitive to oxidative stress $\left(\mathrm{H}_{2} \mathrm{O}_{2}\right)$, cells constitutively equipped to favor the uptake of iron via TfR1 without storing it in Ft, develop sensitivity to $\mathrm{H}_{2} \mathrm{O}_{2}$ through LIP increase (Lipiński et al., 2000). Thus, limiting LIP in mammalian cells seems to be one of the most efficient mechanisms of reducing cell susceptibility to oxidative stress. Several lines of evidence indicate that under oxidative stress, mammalian cells misregulate iron metabolism. Reactive oxygen species influence LIP through the transcriptional and posttranscriptional regulation of ferritin gene and through the release of iron from iron-sulfur proteins and from ferritin degraded in lysosomes (Tsuji et al., 2000).

The question whether reactive oxygen species influence IRP1 RNA-binding activity was addressed in midnineties of the $20^{\text {th }}$ century (Martins et al., 1995; Pantopoulos and Hentze, 1995). An exposure of intact cells to $\mathrm{H}_{2} \mathrm{O}_{2}$ leads to a quick (30 min) activation of IRP1 RNAbinding activity which is followed by an inhibition of ferritin synthesis and a rise in TfR1 mRNA levels (Pantopoulos and Hentze, 1995). Moreover, the perfusion of rat liver with glucose/glucose oxidase/catalase, system that generates steady-state concentrations of $\mathrm{H}_{2} \mathrm{O}_{2}$, leads to a rapid activation of IRP1 IRE-binding activity in the intact organ (Mueller et al., 2001). On the contrary, to what is observed in intact cells, the exposure of cells lysates to $\mathrm{H}_{2} \mathrm{O}_{2}$ as well as an increase in intracellular $\mathrm{H}_{2} \mathrm{O}_{2}$ concentration causes a loss of aconitase activity but does not elicit IRP1 IRE-binding activity (Pantopoulos et al., 1997). Studies using human recombinant IRP1 showed that the aconitase activity of IRP1 is rapidly lost by a reaction with $\mathrm{H}_{2} \mathrm{O}_{2}$ as the [4Fe-4S] cluster is quantitatively converted into the [3Fe-4S] form (which possess neither aconitase nor IRE-binding activity) with the release of a single ferrous ion per molecule (Brazzolotto et al., 1999). Failure of $\mathrm{H}_{2} \mathrm{O}_{2}$ to induce IRE-binding activity of IRP1 in cell extracts led to the proposal that IRP1 activation was not a consequence of a direct action of $\mathrm{H}_{2} \mathrm{O}_{2}$ on the protein and suggested the involvement of $\mathrm{H}_{2} \mathrm{O}_{2}$ induced phosphorylation-dependent signaling pathway (Pantopoulos and Hentze, 1995).

In addition to the inhibition of ferritin mRNA translation via IRP1 activated by exogenous $\mathrm{H}_{2} \mathrm{O}_{2}$, ferritin gene is subjected to transcriptional induction in response to $\mathrm{H}_{2} \mathrm{O}_{2}$ (Torti and Torti, 2002). This former regulation would be expected to negate the transcriptionally mediated increase in ferritin mRNA levels, since the resulting transcripts would not be effectively translated. Regarding the ferritin protein level, it shows an early down-regulation accompanied by an increase in IRP1 IRE-binding activity and delayed up-regulation correlated with deactivation of IRP1 (Tsuji et al., 2000). It has been demonstrated that the relationship between ferritin mRNA, ferritin protein synthesis, and IRP1 activation can be explained by temporal events that occur in response to $\mathrm{H}_{2} \mathrm{O}_{2}$.

The superoxide anion $\left(\mathrm{O}_{2}^{--}\right)$, a product of the oneelectron reduction of $\mathrm{O}_{2}$, is able to directly interact with IRP1 iron-sulfur cluster, and thus, inhibit the aconitase activity of this protein. Evidence obtained mainly from studies using cell-free systems indicates that the reaction of $\mathrm{O}_{2}^{--}$with the IRP1 [4Fe-4S] center does not lead to the formation of an apo-IRP1 able to bind IRE sequences (Bouton et al., 1996). $\mathrm{O}_{2}^{--}$also interacts with the [4Fe-4S] cluster of mitochondrial aconitase and inactivates this Krebs cycle enzyme (Gardner et al., 1995). However, in contrast to the apparent toxicity of this reaction, the biological consequences of $\mathrm{O}_{2}^{--}$-mediated inactivation of the cytosolic counterpart of mitochondrial aconitase, are still not clear.

We have addressed the question whether a deficiency in the superoxide dismutase $1(\mathrm{Cu}, \mathrm{Zn}-\mathrm{SOD})$ in mammals, supposed to increase the $\mathrm{O}_{2}^{--}$steady-state level, modulates IRP1 activities, and, consequently, influences 
iron metabolism. As an experimental model, we used mice lacking activity due to disruption of the Sod1 gene. Apart from the expected decrease in the aconitase activity of IRP1 in $\mathrm{Cu}, \mathrm{Zn}$-SOD the knock-out mice we observed, displayed a marked down-regulation of IRP1 at both mRNA and protein levels. Despite such a major alteration in IRP1 status, iron metabolism appeared to be largely unaffected in $\mathrm{Cu}, \mathrm{Zn}$-SOD-deficient mice, strongly suggesting that IRP1 is not indispensable in the control of iron metabolism under $\mathrm{O}_{2}^{--}$-mediated oxidative stress (Starzyński et al., 2006). It seems reasonable to propose that $\mathrm{O}_{2}^{--}$by decreasing IRP1 level, counteracts the effects of $\mathrm{H}_{2} \mathrm{O}_{2}$-dependent activation of IRP1 and thus serves as a flexible adaptation of iron metabolism to oxidative stress where various ROS are generated. Such bi-directional regulation of IRP1 by oxidants is reminiscent of that mediated by NO.

\section{References}

Andrews N.C. (2008) Forging a field: the golden age of iron biology. Blood 112: 219-230.

Arosio P., Levi S. (2010) Cytosolic and mitochondrial ferritins in the regulation of cellular iron homeostasis and oxidative damage. Biochim. Biophys. Acta. 1800: 783-792.

Bartosz G. (2003) Druga twarz tlenu, Wydawnictwo Naukowe PWN, Warszawa.

Binder R., Horowitz J.A., Basilion J.P., Koeller D.M., Klausner R.D., Harford J.B. (1994) Evidence that the pathway of transferrin receptor mRNA degradation involves an endonucleolytic cleavage within the 3' UTR and does not involve poly(A) tail shortening. EMBO J. 13: 1969-1980.

Bouton C., Chauveau M.J., Lazereg S., Drapier J.C. (2002) Recycling of RNA binding iron regulatory protein 1 into an aconitase after nitric oxide removal depends on mitochondrial ATP. J. Biol. Chem. 277: 31220-31227.

Bouton C., Drapier J.C. (2003) Iron regulatory proteins as NO signal transducers. Sci STKE. 182: pe17.

Bouton C., Oliveira L., Drapier J.C. (1998) Converse modulation of IRP1 and IRP 2 by immunological stimuli in murine RAW 264.7 macrophages. J. Biol. Chem. 273: 9403-9408.

Bouton C., Raveau M., Drapier J.C. (1996) Modulation of iron regulatory protein functions. Further insights into the role of nitrogen- and oxygen-derived reactive species. J. Biol. Chem. 271: 2300-2306.

Brazzolotto X., Gaillard J., Pantopoulos K., Hentze M.W., Moulis J.M. (1999) Human cytoplasmic aconitase (Iron regulatory protein 1) is converted into its [3Fe-4S] form by hydrogen peroxide in vitro but is not activated for iron-responsive element binding. J. Biol. Chem. 274: 21625-21630.

Dandekar T., Stripecke R., Gray N.K., Goossen B., Constable A., Johansson H.E., Hentze M.W. (1991) Identification of a novel iron-responsive element in murine and hu- man erythroid delta-aminolevulinic acid synthase $m R N A$. EMBO J. 10: 1903-1909.

Drapier J.C., Hirling H., Wietzerbin J., Kaldy P., Kühn L.C. (1993) Biosynthesis of nitric oxide activates iron regulatory factor in macrophages. EMBO J. 12: 3643-3649.

Dupuy J., Darnault C., Brazzolotto X., Kühn L.C., Moulis J.M., Volbeda A., Fontecilla-Camps J.C. (2005) Crystallization and preliminary $X$-ray diffraction data for the aconitase form of human iron-regulatory protein 1. Acta Crystallogr. F: Struct. Biol. Cryst. Commun. 61: 482-485.

FeeldersR.A., Kuiper-Kramer E.P.,van Eijk H.G. (1999) Structure, function and clinical significance of transferrin receptors. Clin. Chem. Lab. Med. 37: 1-10.

Galy B., Ferring D., Benesova M., Benes V., Hentze M.W. (2004) Targeted mutagenesis of the murine IRP1 and IRP2 genes reveals context-dependent RNA processing differences in vivo. RNA J10: 1019-1025.

Galy B., Ferring D., Hentze M.W. (2005) Generation of conditional alleles of the murine Iron Regulatory Protein (IRP)1 and -2 genes. Genesis 43: 181-188.

Galy B., Ferring-Appel D., Kaden S., Gröne H.J., Hentze M.W. (2008) Iron regulatory proteins are essential for intestinal function and control key iron absorption molecules in the duodenum. Cell Metab. 7: 79-85.

Galy B., Ferring-Appel D., Sauer S.W., Kaden S., Lyoumi S., Puy H., Kölker S., Gröne H.J., Hentze M.W. (2010) Iron regulatory proteins secure mitochondrial iron sufficiency and function. Cell Metab. 12: 194-201.

Ganz T. (2005) Cellular iron: ferroportin is the only way out. Cell Metab. 1: 155-157.

Gardner P.R.., Raineri I., Epstein L.B., White C.W. (1995) Superoxide radical and iron modulate aconitase activity in mammalian cells. J. Biol. Chem. 270: 13399-13405.

Gunshin H., Mackenzie B., Berger U.V., Gunshin Y., Romero M.F., Boron W.F., Nussberger S., Gollan J.L., Hediger M.A. (1997) Cloning and characterization of a mammalian proton-coupled metal-ion transporter. Nature 388: 482-488.

Guo B., Brown F.M., Phillips J.D., Yu Y., Leibold E.A. (1995) Characterization and expression of iron regulatory protein 2 (IRP2). Presence of multiple IRP2 transcripts regulated by intracellular iron levels. J. Biol. Chem. 270: 16529-16535.

Guo B., Yu Y., Leibold E.A. (1994) Iron regulates cytoplasmic levels of a novel iron-responsive element-binding protein without aconitase activity. J. Biol. Chem. 269: 2425224260.

Hanson E.S., Rawlins M.L., Leibold E.A. (2003) Oxygen and iron regulation of iron regulatory protein 2.J. Biol. Chem. 278: 40337-40342.

Henderson B.R., Menotti E., Bonnard C., Kühn L.C. (1994) Optimal sequence and structure of iron-responsive elements. Selection of RNA stem-loops with high affinity for iron regulatory factor. J. Biol. Chem. 269: 17481-17489.

Hentze M.W., Muckenthaler M.U., Galy B., Camaschella C. (2010) Two to tango: regulation of Mammalian iron metabolism. Cell. 142: 24-38. 
Hirling H., Henderson B.R., Kühn L.C. (1994) Mutational analysis of the [4Fe-4S]-cluster converting iron regulatory factor from its RNA-binding form to cytoplasmic aconitase. EMBO J. 13: 453-461.

Ishikawa H., Kato M., Hori H., Ishimori K., Kirisako T., Tokunaga F., Iwai K. (2005) Involvement of heme regulatory motif in heme-mediated ubiquitination and degradation of IRP2. Mol. Cell. 19: 171-181.

Kakhlon O., Cabantchik Z.I. (2002) The labile iron pool: characterization, measurement, and participation in cellular processes(1). Free Radic. Biol. Med. 33: 1037-1046.

Kim S., Wing S.S., Ponka P. (2004) S-nitrosylation of IRP2 regulates its stability via the ubiquitin-proteasome pathway. Mol. Cell Biol. 24: 330-337.

Kohler S.A., Henderson B.R., Kühn L.C. (1995) Succinate dehydrogenase $b \mathrm{mRNA}$ of Drosophila melanogaster has a functional iron-responsive element in its 5'-untranslated region. J. Biol. Chem. 270: 30781-30786.

Kruszewski M. (2003) Labile iron pool: the main determinant of cellular response to oxidative stress. Mutat. Res. 531: 81-92.

LaVaute T., Smith S., Cooperman S., Iwai K., Land W., Meyron-Holtz E., Drake S.K., Miller G., Abu-Asab M., Tsokos M. et al. (2001) Targeted deletion of the gene encoding iron regulatory protein-2 causes misregulation of iron metabolism and neurodegenerative disease in mice. Nat. Genet. 27: 209-214.

Leibold E.A., Munro H.N. (1988) Cytoplasmic protein binds in vitro to a highly conserved sequence in the 5 'untranslated region of ferritin heavy- and light-subunit mRNAs. Proc. Natl. Acad. Sci. USA 85: 2171-2175.

Lipiński P., Drapier J.C., Oliveira L., Retmańska H., Sochanowicz B., Kruszewski M. (2000) Intracellular iron status as a hallmark of mammalian cell susceptibility to oxidative stress: a study of L5178Y mouse lymphoma cell lines differentially sensitive to $\mathrm{H}_{2} \mathrm{O}_{2}$. Blood 95: 2960-2966.

Lipiński P., Starzyński R.R. (2006) The role of iron regulatory proteins (IRPS) in the regulation of systemic iron homeostasis: lessons from studies on IRP1 and IRP2 knock out mice. Postepy Hig. Med. Dosw. 60: 322-330.

Lipiński P., Starzyński R.R., Drapier J.C., Bouton C., Bartłomiejczyk T., Sochanowicz B., Smuda E., Gajkowska A., Kruszewski M. (2005) Induction of iron regulatory protein $1 R N A$ binding activity by nitric oxide is associated with a concomitant increase in the labile iron pool: implications for DNA damage. Biochem. Biophys. Res. Commun. 327: 349-355.

Lipiński P., Starzyński R.R., Styś A., Straciło M. (2010) Iron homeostasis, a defense mechanism in oxidative stress. Post. Biochem. 56: 305-316.

Martins E.A., Robalinho R.L., Meneghini R. (1995) Oxidative stress induces activation of a cytosolic protein responsible for control of iron uptake. Acta Biochem. Biophys, 316: 128-134.

Meyron-Holtz E.G., Ghosh M.C., Iwai K., LaVaute T., Brazzolotto X., Berger U.V., Land W., Ollivierre-Wilson H., Grinberg A., Love P. et al. (2004) Genetic ablations of iron re- gulatory proteins 1 and 2 reveal why iron regulatory protein 2 dominates iron homeostasis. EMBO J. 23: 386-395.

Muckenthaler M.U., Galy B., Hentze M.W. (2008) Systemic iron homeostasis and the iron-responsive element/ironregulatory protein (IRE/IRP) regulatory network. Ann. Rev. Nut. 28: 197-213.

Muckenthaler M., Gray N.K., Hentze M.W. (1998) IRP-1 binding to ferritin $m R N A$ prevents the recruitment of the smallribosomalsubunit by the cap-binding complex eIF4F. Mol. Cell 2: 383-388.

Mueller S., Pantopoulos K., Hübner C.A., Stremmel W., Hentze M.W. (2001) IRP1 activation by extracellular oxidative stress in the perfused rat liver. J. Biol. Chem. 76: 23192-23196.

Mulero V., Brock J.H. (1999) Regulation of iron metabolism in murine J774 macrophages: role of nitric oxide-dependent and -independent pathways following activation with gamma interferon and lipopolysaccharide. Blood 94: 23832389.

Mullner E.W., Kuhn L.C. (1988) A stem-loop in the 3' untranslated region mediates iron-dependent regulation of transferrin receptor $m R N A$ stability in the cytoplasm. Cell 53: $815-825$.

Nagy A. (2000) Cre recombinase: the universal reagent for genome tailoring. Genesis. 26: 99-109.

Oliveira L., Drapier J.C.. (2000) Down-regulation of iron regulatory protein 1 gene expression by nitric oxide. Proc. Natl Acad. Sci. USA 97: 6550-6555.

Pantopoulos K., Hentze M.W. (1995) Rapid responses to oxidative stress mediated by iron regulatory protein. $\mathrm{EMBO}$ J., 14: 2917-2924.

Pantopoulos K., Mueller S., Atzberger A., Ansorge W., Stremmel W., Hentze M.W. (1997) Differences in the regulation of iron regulatory protein-1 (IRP-1) by extra- and intracellular oxidative stress. J. Biol. Chem. 272: 9802-9808.

Phillips J.D., Kinikini D.V., Yu Y., Guo B., Leibold E.A. (1996) Differential regulation of IRP1 and IRP2 by nitric oxide in rat hepatoma cells. Blood 87: 2983-2992.

Radisky D.C., Kaplan J. (1998) Iron in cystolic ferritin can be recycled through lysosomal degradation in human fibroblasts. Biochem. J. 15: 201-205.

Recalcati S., Taramelli D., Conte D., Cairo G. (1998) Nitric oxide-mediated induction of ferritin synthesis in J774 macrophages by inflammatory cytokines: role of selective iron regulatory protein-2 downregulation. Blood 91: 10591066.

Rouault T.A. (2006) The role of iron regulatory proteins in mammalian iron homeostasis and disease. Nat. Chem. Biol. 2: 406-414.

Salahudeen A.A., Thompson J.W., Ruiz J.C., Ma H.W., Kinch L.N., Li Q., Grishin N.V., Bruick R.K. (2009) AnE3 ligase possessing an iron-responsive hemerythrin domain is a regulator of iron homeostasis. Science 326: 722-726.

Samaniego F., Chin J., Iwai K., Rouault T.A., Klausner R.D. (1994) Molecular characterization of a second iron-responsive element binding protein, iron regulatory protein 
2. Structure, function, and post-translational regulation. J. Biol. Chem. 269: 30904-30910.

Schalinske K.L., Blemings K.P., Steffen D.W., Chen O.S., Eisenstein R.S. (1997) Iron regulatory protein 1 is not required for the modulation of ferritin and transferrin receptor expression by iron in a murine pro-B lymphocyte cell line. Proc. Nat. Acad. Sci. USA 94: 10681-10686.

Shi H., Bencze K.Z., Stemmler T.L., Philpott C.C. (2008) $A$ cytosolic iron chaperone that delivers iron to ferritin. Science 320: 1207-1210.

Smith S.R., Cooperman S., LaVaute T., Tresser N., Ghosh M., Meyron-Holtz E., Land W., Ollivierre H., Jortner B., Switzer R. $3^{\text {rd }}$ et al. (2004) Severity of neurodegeneration correlates with compromise of iron metabolism in mice with iron regulatory protein deficiencies. Ann. N. Y. Acad. Sci. 1012: 65-83.

Smith S.R., Ghosh M.C., Ollivierre-Wilson H., Hang Tong W., Rouault T.A. (2006) Complete loss of iron regulatory proteins 1 and 2 prevents viability of murine zygotes beyond the blastocyst stage of embryonic development. Blood Cells Mol. Dis. 36: 283-287.

Soum E., Drapier J.C. (2003) Nitric oxide and peroxynitrite promote complete disruption of the $[4 \mathrm{Fe}-4 \mathrm{~S}]$ cluster of recombinant human iron regulatory protein 1. J. Biol. Inorg. Chem. 8: 226-232.

Starzyński R.R., Lipiński P., Drapier J.C., Diet A., Smuda E., Bartlomiejczyk T., Gralak M.A., Kruszewski M. (2005) Down-regulation of iron regulatory protein 1 activities and expression in superoxide dismutase 1 knock-out mice is not associated with alterations in iron metabolism. J. Biol. Chem. 280: 4207-4212.

Starzyński R.R., Gonçalves A.S., Muzeau F., Tyrolczyk Z., Smuda E., Drapier J.C., Beaumont C., Lipiński P. (2006) STAT5 proteins are involved in down-regulation of iron regulatory protein 1 gene expression by nitric oxide. Biochem. J. 400: 367-375.

Styś A., Galy B., Hentze M.W., Bouton C., Lipiński P. (2010) IRP1 but not IRP2 mediates the regulation of intracellular iron metabolism in response to nitric oxide. European Iron Club Meeting 2010, Nijmegen, September $15-17^{\text {th }}$ 2010: 17 .
Torti F.M., Torti S.V. (2002) Regulation of ferritin genes and protein. Blood 99: 3505-3516.

Tsuji Y., Ayaki H., Whitman S.P., Morrow C., Torti S.V., Torti F.M. (2000) Coordinate transcriptional and translational regulation of ferritin in response to oxidative stress. Mol. Cell Biol. 20: 5818-5827.

Vashisht A.A., Zumbrennen K.B., Huang X., Powers D.N., Durazo A., Sun D., Bhaskaran N., Persson A., Uhlen M., Sangfelt O. et al. 2009. Control of iron homeostasis by an iron-regulated ubiquitin ligase. Science 326: 718-721.

Viatte L., Vaulont S. (2009) Hepcidin, the iron watcher. Biochimie. 91: 1223-1228.

Volz K. (2008) The functional duality of iron regulatory protein 1. Curr. Opin. Struct. Biol. 18: 106-111.

Wallander M.L., Leibold E.A., Eisenstein R.S. (2006) Molecular control of vertebrate iron homeostasis by iron regulatory proteins. Biochim. Biophys. Acta. 1763: 668-689.

Wang J., Chen G., Pantopoulos K. (2005) Nitric oxide inhibits the degradation of IRP2. Mol. Cell Biol. 25: 1347-1353.

Watts R.N., Ponka P., Richardson D.R. (2003) Effects of nitrogen monoxide and carbon monoxide on molecular and cellular iron metabolism: mirror-image effector molecules that target iron. Biochem J. 369: 429-440.

Weinberg E.D. (1997) The Lactobacillus anomaly: total iron abstinence. Perspect. Biol. Med. 40: 578-583.

Weiss G., Goossen B., Doppler W., Fuchs D., Pantopoulos K., Werner-Felmayer G., Wachter H., Hentze M.W. (1993) Translational regulation via iron-responsive elements by the nitric oxide/NO-synthase pathway. EMBO J. 12: 36513657.

Ye H., Rouault T.A. (2010) Human iron-sulfur cluster assembly, cellular iron homeostasis, and disease. Biochemistry 49: 4945-4456.

Zheng L., Kennedy M.C., Blondin G.A., Beinert H., Zalkin H. (1992) Purification and characterization of cytosolic aconitase from beef liver and its relationship to the iron-responsive element binding protein. Arch. Biochem. Biophys. 299: 356-360. 